\title{
Changes of Blood Pressure by Wellness Fasting from Chronomedical Aspect
}

\author{
Izumi Utada ${ }^{1}$, Masahito Takahashi ${ }^{2}$, Shaw Watanabe2*, Tanji Hoshi ${ }^{3}$, Satoko Hitomi ${ }^{1}$ and Reiko Takiguchi ${ }^{1}$ \\ ${ }^{1}$ ABO Group ARSOA Co. Ltd., Japan \\ ${ }^{2}$ Life Science Promoting Association, Asia Pacific Clinical Nutrition Society, Japan \\ ${ }^{3}$ Tokyo Metropolitan University, Japan \\ *Corresponding Author: Shaw Watanabe, Life Science Promoting Association, Asia Pacific Clinical Nutrition Society, Japan.
}

Received: July 08, 2019; Published: July 30, 2019

DOI: 10.31080/ASNH.2019.03.0385

\begin{abstract}
Hypertension is becoming pandemic and WHO estimated the prevalence rate of hypertension being one-third of people aged above 25 years old. Number of patients with hypertension in Japan increased from 9,067,000 in 2012 to 10,108,000 in 2015, and its medical cost was 1,889 billion yen. Number of annual death was 6,932, so the prevention of hypertension is an urgent issue, because of its high risk for cardiovascular disease, cerebral palsy, renal insufficiency, and so forth.

We found that suitable fasting is effective in maintaining and/or promoting health, and hypoglycemia by fasting yielded a high concentration of acetone body, especially $\beta$-hydoxybutyrate. We also found systolic and diastolic blood pressure decreased during fasting and its effects continued at least one month later. It seemed to be a legacy effect of the wellness fasting, so we analyzed the factors and considered the mechanism of this phenomenon.
\end{abstract}

Keywords: Hypertension; Fasting; B-Hydoxybutyrate

\section{Introduction}

The importance of ketone body metabolism in diabetic patients has been well known since the 1970s, but recently, $\beta$-hydroxybutyrate (BHB) has been identified as a key component of a metabolic signaling pathway [1-5]. Recently the relationship between fasting and circadian rhythm has been reported [6]. The bidirectional relationship between circadian disruption and metabolic abnormalities is found in diabetic patients. The circadian rhythm in food intake should be strongly affected by fasting. When glucose function changes, circadian behavior should be changed.

We have practiced Kushi macrobiotics for more than 10 years and developed the teaching and practicing system of a 4-day "Wellness-fasting" [7-9]. Our previous study in wellness fasting and BHB production by PASS analysis showed that the decreased BMR and insulin significantly contributed to the increase in BHB and acetyl acetate (AcAc) [9]. In this paper, we would like to focus on the changes in blood pressure in conjunction with other physical markers during wellness fasting.

\section{Subjects and Methods}

In ARSOA Wellness Fasting 4 days program, participants received a combination of the very low-energy vegetarian diet, physical exercise, meditation, and lectures about healthy lifestyles
[7,9]. (Figure 1) Data were collected from October 2014 to October 2018. Inclusion criteria to participate in the program were healthy adults in any age in both sex, and exclusion criteria were diseased patients receiving drug therapy for cardiovascular disease, diabetes, chronic infectious diseases, gastrointestinal (GI) tract diseases, cancer, and pregnant women. All participants signed the agreement sheet after a precise explanation of the program. The program had been approved by the Ethical Committee in the Life Science Promotion Association.

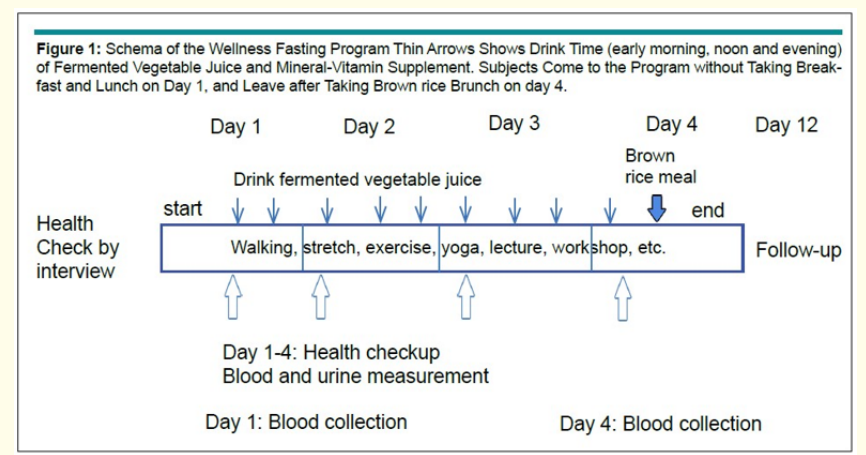

Figure 1: Schema of 4-day wellness fasting. Daily energy intake is about $350 \mathrm{kcal}$ from the fermented vegetable juice. 
Peripheral blood was collected on days 1 and 4 for routine biochemical analyses and for the determination of insulin serum levels, glucagon, and other hormones. Glucose and BHB were measured every morning by the finger-tip blood. The participants registered at noon on the first day. After check-in, health check-up was done by the Body Composition Analyzer (Tanita, Tokyo, Japan) which measured body weight, fat volume, muscle volume, water contents, estimated bone weight, basal metabolic rate and body mass index (BMI), based on the electric impedance. Blood pressure and pulse rate were measured by Omron HEM-7021 (Kyoto, Japan). Each participant was requested to fulfill the health checkup sheet, including subjective psychological symptoms by 3 categories (not feel, feel, strongly feel), such as headache, hungry, GI distress, nausea, fatigue, disgust or depression, general condition, vivid feeling, activity, and skin condition.

\section{Statistical Analysis}

All data were collected in the Excel database and transferred to SPSS version 20 (IBM SPSS, Tokyo, Japan). Non-parametric variables, such as mood, were replaced to categorical variables. Significant level was set at $\mathrm{p}<0.05$. We also used structural equation modeling (SEM) to examine presumptive underlying variables by AMOS version 19 for Windows (IBM Inc.). The analysis was performed using cross-lagged effects variation model with longitudinal data followed over one month. Estimation of the best fitting model was carried out by the method of maximum likelihood of SEM. Results were regarded as statistically significant if the $p$ values were less than 0.05 .

\section{Results}

The total number of participants to the program was $233 ; 44$ males and 189 females (Table 1 ). The mean age was $43.9+/-9.2$ years old in males and $46.6+/-12.5$ in females. Body weight was $63.6+/-2.6 \mathrm{~kg}$ in males and 55.4+/- 3.6 in females.

\begin{tabular}{|c|c|c|c|}
\hline & Male & Female & Total \\
\hline 20 's & 2 & 26 & 28 \\
\hline 30 's & 16 & 27 & 43 \\
\hline 40 's & 16 & 54 & 70 \\
\hline 50 's & 8 & 54 & 62 \\
\hline 60 's & 1 & 24 & 25 \\
\hline 70 's & 1 & 4 & 5 \\
\hline & 44 & 189 & 233 \\
\hline
\end{tabular}

Table 1: Number of participants by sex and age category.

BHB in the blood increased from $0.3 \pm 0.2 \mathrm{mM}$ at the day 1 to 2.0 $\pm 1.2 \mathrm{mM}$ at the day 4 of fasting, while the glucose level decreased from $5.6 \pm 1.6 \mathrm{mM}$ to $3.9 \pm 1.3 \mathrm{mM}$ at 4 day (Table 2). According to the increase of blood ketone bodies, urinary excretion of median acetylacetate increased to $40.9 \mathrm{mM}$ (7.76-159.2) (min-max), BHB 25.1 mM (2.72-597.0) and total ketone bodies $66.1 \mathrm{mM} \mathrm{(10.5-}$ $756.5)$ on the 4 th day. Individual difference of BHB increase was present (Figure 2). These values returned to $0.16 \mathrm{mM}(0-10.1)$ on the 14 day (10 days after fasting).

\begin{tabular}{|l|l|l|l|}
\hline & \multicolumn{2}{|c|}{ glucose } & \multicolumn{2}{c|}{$\mathrm{bHB}$} \\
\hline Day1 & $5.0 \pm$ & 0.9 & $0.3 \pm 0.2$ \\
\hline Day2 & $4.8 \pm$ & \pm .6 & $0.4 \pm 0.3$ \\
\hline Day3 & $4.2 \pm$ & \pm .7 & $1.1 \pm 0.7$ \\
\hline Day4 & $3.9 \pm$ & \pm .8 & $1.9 \pm 1.2$ \\
\hline
\end{tabular}

Table 2: Changes in glucose and BHB during 4-day fasting.

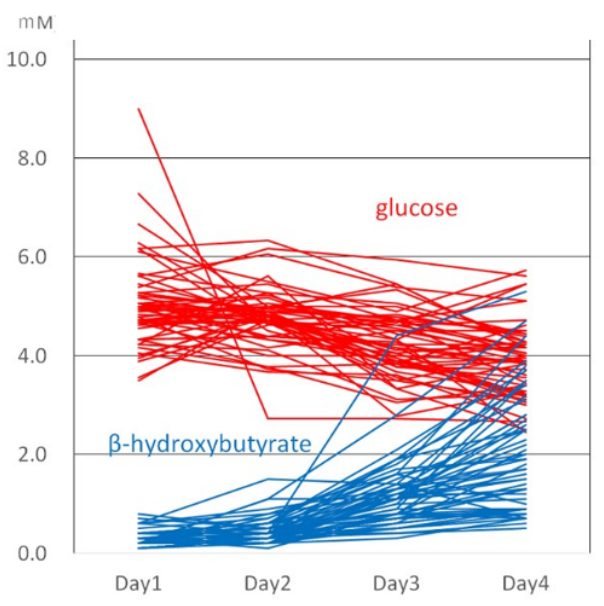

Figure 2: Individual change of blood glucose and BHB level.

Although headaches, hunger, GI tract distress, emesis, depression, and cold feeling occurred toward the $2^{\text {nd }}$ day of fasting, these symptoms dramatically disappeared at the $3^{\text {rd }}$ day, and active and vivid feeling increased on the 4th day and after. Headache was a common complaint in females but their improvement is better than that of males by fasting.

BMI was $24.3 \pm 2.3$ in males and $21.8 \pm 3.3$ in females on the first day (Table 3). Body weight decreased from $55 \mathrm{~kg}$ to $53.5 \mathrm{~kg}$ in females, and it continued at least one month later. Waist circumference decreased from $85 \mathrm{~cm}$ to $82 \mathrm{~cm}$ on day 4 , and it also continued for one month. Body weight reduction was $2.5 \mathrm{~kg}$ in males and 1.7 $\mathrm{kg}$ in females. Body fat rate decreased by $0.8 \%$ and $0.7 \%$ in males and females, respectively, and muscular weight decreased $1.3 \mathrm{~kg}$ in males and $0.8 \mathrm{~kg}$ in females.

\begin{tabular}{|c|c|c|c|c|c|c|c|c|}
\hline & \multicolumn{4}{|c|}{ Males $n=44$} & \multicolumn{4}{|c|}{ Females $n=189$} \\
\hline & day & & day 4 & difference & & ay 1 & day 4 & difference \\
\hline & mean & sd & & & mean & sd & & \\
\hline body weight & 67.4 & \pm 8.1 & $64.4 \pm 7.4$ & $2.5 \pm 0.7$ wow & 550 & \pm 8.4 & $53.2 \pm 8.0$ & $1.7 \pm 0.9$ *w* \\
\hline BMI & 24.3 & \pm 23 & $23.3 \pm 2.2$ & $1.0 \pm 0.3 \ldots$ & 21.8 & $\begin{array}{l} \pm 3.3 \\
\pm 3\end{array}$ & $21.2 \pm 3.3$ & $0.6 \pm 0.5 \mathrm{k} *$ \\
\hline FAT\% & 21.4 & \pm 4.7 & $20.4 \pm 4.4$ & $0.8 \pm 1.3$ *wok & 30.8 & \pm 6.2 & $30.0 \pm 6.7$ & $0.7 \pm 2.4$ kack \\
\hline MUSCLE & 50.0 & \pm 3.9 & $48.4 \pm 3.9$ & $1.3 \pm 0.8$ *atk & 35.4 & \pm 3.2 & $34.6 \pm 3.1$ & $0.8 \pm 1.3$ *awk \\
\hline WATER\% & 36.2 & \pm 2.7 & $34.7 \pm 2.2$ & $1.2 \pm 0.9 *$ & 25.7 & \pm 2.8 & $25.4 \pm 3.0$ & $0.4 \pm 0.9 *$ \\
\hline BMR & 1567.0 & \pm 23.4 & $1425.5 \pm 94.9$ & $41.7 \pm 21.4 \times \mathrm{kat}$ & 1134.0 & \pm 1390 & $1113.0 \pm 1340$ & $22.6 \pm 32.4 \times \mathrm{k}$ \\
\hline
\end{tabular}

Table 3: Changes of body composition by 4-day wellness fasting.

Blood pressure during fasting continuously decreased (Table 4).

Systolic blood pressure decreased $12 \mathrm{mmHg}$ in both males and females, and $5 \mathrm{mmHg}$ and $6 \mathrm{mmHg}$ in diastolic pressure in males and females, respectively. On the contrary, the median pulse rate increased $10 / \mathrm{min}$ in males and $7.5 / \mathrm{min}$ in females. Body temperature decreased $0.2-0.3 \mathrm{C}$ in both sex (Figure 3). 


\begin{tabular}{|lrrrr|}
\hline & \multicolumn{2}{c}{$M(\mathrm{n}=43)$} & \multicolumn{2}{c|}{$\mathrm{F}(\mathrm{n}=1 \mathbf{8 6})$} \\
\hline & average & \multicolumn{1}{c}{ sd } & average & \multicolumn{1}{c|}{ sd } \\
\hline age & 43.9 & \multicolumn{1}{c}{9.2} & 47.2 & 12.5 \\
\hline sysbp1 & $\mathbf{1 3 0 . 6}$ & 15.7 & $\mathbf{1 2 2 . 9}$ & 21 \\
sysbp2 & $\mathbf{1 2 1 . 1}$ & 15.3 & $\mathbf{1 1 5 . 1}$ & 17.5 \\
sysbp3 & $\mathbf{1 1 7 . 9}$ & 10.5 & $\mathbf{1 0 9 . 8}$ & 16 \\
sysbp4 & $\mathbf{1 1 6 . 4}$ & 8.5 & $\mathbf{1 0 8 . 8}$ & 13.7 \\
sysb1 $\mathrm{w}$ & 122.2 & 12.7 & 115 & 14.8 \\
sysbp1 $\mathrm{m}$ & 123 & 9 & 119.6 & 14.3 \\
\hline diabp1 & $\mathbf{8 7 . 1}$ & 12.9 & $\mathbf{8 1 . 4}$ & 13 \\
diabp2 & $\mathbf{8 2 . 3}$ & 11.4 & $\mathbf{7 7 . 7}$ & 11.3 \\
diabp3 & $\mathbf{7 9 . 7}$ & 10.1 & $\mathbf{7 5 . 3}$ & 10.7 \\
diabp4 & $\mathbf{8 0 . 4}$ & 8.2 & $\mathbf{7 5 . 7}$ & 10 \\
diabp1 $\mathrm{w}$ & $\mathbf{7 7 . 7}$ & 8.7 & $\mathbf{7 5 . 9}$ & 11.4 \\
diabp1 $\mathrm{m}$ & 82 & 7.9 & $\mathbf{7 5 . 6}$ & 9.6 \\
\hline puls1 & $\mathbf{7 3 . 8}$ & 12.8 & $\mathbf{7 4 . 4}$ & 11.1 \\
puls2 & $\mathbf{7 5 . 3}$ & 14.1 & $\mathbf{7 6 . 8}$ & 10.2 \\
puls3 & $\mathbf{8 0 . 7}$ & 14.1 & $\mathbf{7 9 . 7}$ & 11.7 \\
puls4 & $\mathbf{8 3 . 7}$ & 10.6 & $\mathbf{8 1 . 7}$ & 10.5 \\
puls1 $\mathrm{w}$ & 64.3 & 7.8 & $\mathbf{7 2 . 3}$ & 9.6 \\
puls1 $\mathrm{m}$ & 67.4 & 13.2 & $\mathbf{7 0 . 5}$ & 9.6 \\
\hline
\end{tabular}

Table 4: Changes of blood pressure and pulse rate during 4-day fasting and 1 month later Sysbp; systolic blood pressure at 1,2,3,4 day, 1 week and 1month later, diabp; diastolic blood pressure, puls; pulse rate at the same interval.
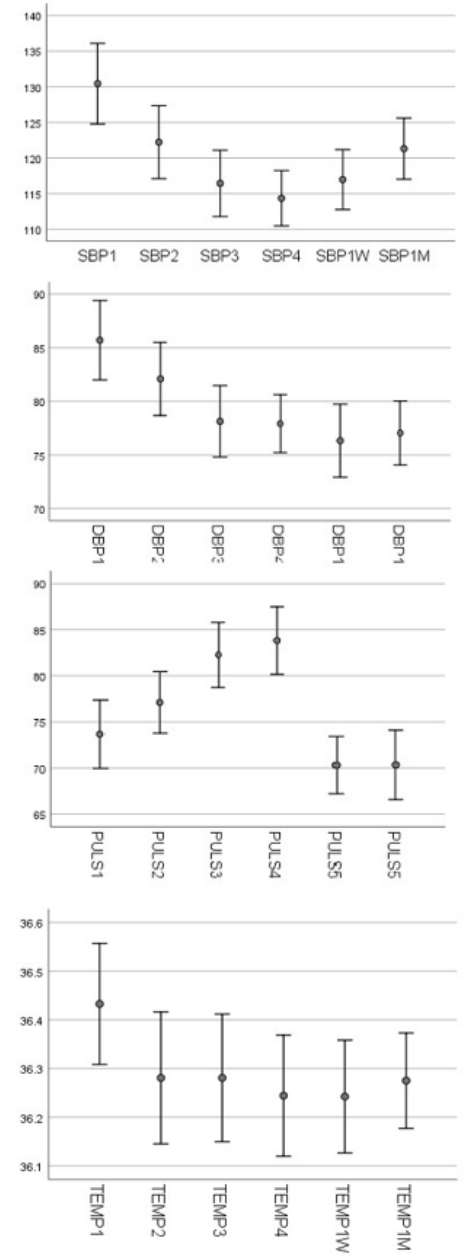

Figure 3: Changes in blood pressure, pulse rate, and body temperature during 4-day fasting and after 1 week $(1 \mathrm{~W})$ and 1 month (1M). SBP; systolic blood pressure, DBP; diastolic blood pressure, PULS; pulse rate, TEMP; body temperature.
By correlation analysis, BHB difference only correlated to the body temperature and pulse rate, and glucose decrease correlated with lowered diastolic pressure (Table 5).

\begin{tabular}{|c|c|c|c|c|c|c|c|c|c|}
\hline & , & Dintuan? 4 & & & difi1 & IRdif & Sulft1_.4 & Ediff & \\
\hline tempdif1_4 $\mathrm{P}^{\prime} \mathrm{CO}$ & 1 & -0.02 & -0 & 0.00 & 0.17 & 0.011 & -0.07 & -0.037 & $.193^{* *}$ \\
\hline & & 0.90 & 0.78 & 0.98 & 0.23 & 0.937 & 0.34 & 0.598 & 0.008 \\
\hline bmidif1_4 $F^{\prime} \mathrm{CO}$ & -0.02 & 1.00 & -0.1 & $.639 * *$ & $.358 * *$ & $.796 * *$ & 0.04 & 0.048 & -0.106 \\
\hline$D$ & 0.903 & & 0.6 & 0.00 & 0.01 & 0 & 0.73 & 0.693 & 0.411 \\
\hline bfatdif1 $4 \quad F^{\prime} C O$ & -0.02 & -0.06 & & $-0.695^{* *}$ & $-0.57 k *$ & $-.546 * *$ & 0.01 & 0.083 & -0.037 \\
\hline & 0.778 & 0.60 & & 0.00 & 0.00 & 0 & 0 & 0.232 & 0.613 \\
\hline musdif1 & & $.639 * *$ & -.695 & 1.00 & $.697 * *$ & $.958^{* * *}$ & 0.04 & -0.051 & -0.001 \\
\hline & 0.984 & 0.00 & & & 0.00 & 0 & 0.54 & 0.472 & 0.992 \\
\hline watdif1 ${ }^{4} \quad \mathrm{P}^{\prime} \mathrm{CO}$ & 0.168 & $.358 * *$ & -.570 & $.697 * *$ & 1.00 & $.673^{* *}$ & 0.02 & -0.024 & -0.212 \\
\hline p & 0.229 & 0.01 & 0 & 0.00 & & .070 & 088 & 0.86 & 0.148 \\
\hline bmrdif1 _4 $P^{\prime} \mathrm{CO}$ & 0.011 & $796 * *$ & -.546 & $958^{* *}$ & $673^{* *}$ & 1 & 9 & -0.003 & -0.057 \\
\hline & 0.937 & 0.00 & 0 & 0.00 & 0.00 & & 0.52 & 0.985 & 0.701 \\
\hline sysdiff1_4 & -0 & & 0.0 & & 0.02 & 0.089 & 1.00 & $.647 * *$ & 0.056 \\
\hline & $a_{3}$ & & 0. & 0. & 0.88 & 0.517 & & & 0.442 \\
\hline diadiff1_4 & -0.04 & 0.05 & 0.08 & -0.05 & -0.02 & -0.003 & 3.647** & & $.224 * *$ \\
\hline & 0.598 & 0.69 & 0.23 & 0.47 & 0.86 & 0.985 & 0.00 & & 0.002 \\
\hline pulsdiff1 $4 \mathrm{~F}^{\prime} \mathrm{O}$ & $.193^{* *}$ & -0.11 & -0 & 0.00 & -0.21 & -0.057 & 0.06 & $.224 * *$ & 1 \\
\hline & 0.008 & 0.41 & 0.61 & 0.99 & 0.15 & 0.701 & 0.44 & 0.002 & \\
\hline
\end{tabular}

Table 5: Correlation analysis between changes of body composition and circulatory index

Tempdf; difference of temperature between the $1^{\text {st }}$ and $4^{\text {th }}$ day, bmidif; difference of BMI, dfat; difference of fat \%, musdif; difference of muscle volume, watdif; difference of water \%, bmrdif; difference of BMR, sysdiff; difference of systolic blood pressure, diadiff; difference of diastolic blood pressure, pulsidff; difference of pulse rate.

Changes in blood pressure and pulse rate were influenced by age category (Figure 4).

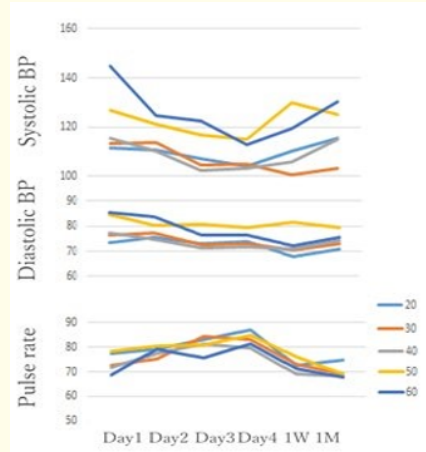

Figure 4: Changes in blood pressure and pulse rate by age category.

Older age group more than age 60 years was hypertensive on day 1 , although the pulse rate was lowest among the age groups, it improved rapidly toward the 4th day in both systolic and diastolic blood pressure. Even after one month, it remained within the normal range. On the contrary, increased pulse rate rapidly returned to the $70 / \mathrm{min}$ by stopping fasting.

Chief component analysis to find factors of these changes by further analysis could extract 3 axis (Table 6). 


\begin{tabular}{|l|r|r|r|}
\hline & 1 & 2 & 3 \\
\hline BMR1 M & 0.995 & 0.07 & 0.062 \\
\cline { 2 - 3 } WATER4\% & 0.769 & 0.025 & 0.302 \\
\cline { 2 - 3 } BFAT1 M\% & 0.683 & 0.195 & -0.263 \\
\cline { 1 - 3 } SBP1 M & 0.146 & 0.987 & 0.055 \\
DBP1 M & -0.095 & 0.769 & 0.19 \\
\hline TEMP1 M & -0.079 & -0.104 & -0.018 \\
\hline MUSCLE1 & 0.314 & 0.013 & 0.949 \\
\hline PULS5 & 0.204 & -0.182 & -0.401 \\
\hline FEEL1 M & 0.149 & 0.232 & 0.361 \\
\hline VIVID1 M & 0.161 & 0.313 & -0.337 \\
\hline
\end{tabular}

Table 6: Chief component analysis of biomarkers

BMR1M; BMR at 1month after fasting, WATER4\%, body water $\%$ at the 4 th day, BFAT1M\%; body fat composition at 1 month, SBP1M; systolic blood pressure at 1 month, DBP1M; diastolic blood pressure at 1 month, TEMP1M; Body temperature at 1 month, MUSCLE1M; muscle volume $\%$ at 1 month, PULSE5; pulse rate at 4th day, FEEL1M; feeling at 1 month, VIVID1M; vivid feeling at 1 month.

The structural equation modeling (SEM) to examine presumptive underlying variables by AMOS. The latent variable "BP1M" associated with systolic blood pressure and diastolic blood pressure one month after fasting was directly defined as the blood pressure "BP 1" on the first day of fasting and from the normalized estimated value of 0.90 under 49 years of age directly.

Even over 50 years old, the standardization estimate was 0.54 and it was a high expectation (Figure 5). From the pulse rate "PLSE 234 " to "BP $1 \mathrm{M}$ " on the $2^{\text {nd }}, 3^{\text {rd }}$, and $4^{\text {th }}$ fasting days, the standardized estimated value of -0.36 was found directly below the age of 49 , indicating a direct effect of lowering the blood pressure. However, at the age of over 50 years, it increased +0.40 and "BP $1 \mathrm{M}$ " rather. However, over the age of 50 years, from "PLSE 234", through the reduction of "BODY 4" on the fourth day of fasting related to BMI and muscle and water, the overall effect on "BP $1 \mathrm{M}$ " is $-0.46 \times 0.41$. It was shown that blood pressure of 0.19 and one month was decreased by the total effect.
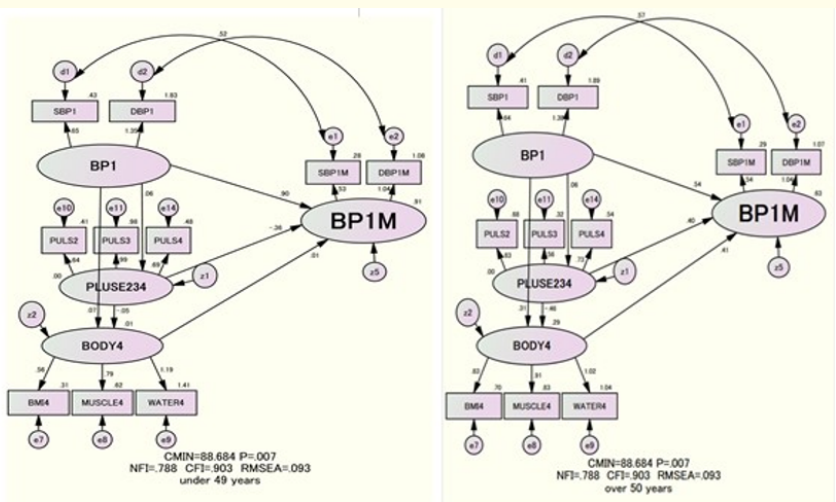

Figure 5: The structural equation modeling by AMOS.

Legacy effects of blood pressure one month later could be explained by the combined blood pressure of Day1, pulse rate and body composition of the Day 4 . The contribution of pulse rate

is reversed under 49 years of age and over 50 years of age.
The causal structure in which blood pressure stabilized through physical condition was high fitness degree, in which $63 \%$ can be explained. However, in the stabilization of blood pressure one month later, different mechanisms were observed depending on age. Gender analysis is planned to be analyzed after increasing the number of cases.

According to this model, 1-month blood pressure, BPM1, under 49 years old could explain $91 \%$, and 50 years old or older the fitness of this model is NFI $=0.788, \mathrm{CFI}=0.903$, and RMSEA $=0.093$, so a significantly high degree of conformity was obtained.

\section{Discussion}

Current global mortality from noncommunicable diseases (NCDs) remains unacceptably high. Thirty-eight million people die each year from NCDs, mainly from cardiovascular diseases, cancers, chronic respiratory diseases, and diabetes. Over 14 million deaths from NCDs occur between the ages of 30 and 70, of which $85 \%$ are in developing countries. These premature deaths are largely preventable by implementing simple measures which reduce risk factors for NCDs. Hypertension is the largest preclinical basis of cardiovascular diseases and could be controlled by changes in life habits.

So far, the low sodium diet has been recommended to improve hypertension. The decrease of blood pressure by fasting was an impact. We have supported basically macrobiotics, which was elaborated to take the balance of food, physical activity and keeping a mind to achieve a spiritual life. It is relevant to the Koda's fasting therapy [10], in which unpolished brown rice and green vegetable paste constitute the basic regimen, and it improves the intestinal environment by resolving constipation. Koda's method could repair the balance of the autonomic nervous system, so it may not only be effective for gastrointestinal disease, but also for some neurodegenerative diseases.

Four days fasting-induced hyperketosis without concurrent clinical symptom. The magnitude of changes in ketone body concentrations correlates with the levels of insulin, glucose, free fatty acids, and several hormones [9]. Very stable glucose levels, even though at the hypoglycemic level, were noteworthy under ketogenic change during fasting. Fasting caused hyperketonemia, but the degree was different by individuals. Enhanced ketone body production occurred when the blood glucose level was less than $4.5 \mathrm{mM}$ $(90 \mathrm{mg} / \mathrm{dl})$. The urinary excretion of BHB/acetylacetonate (AcAc) ratio increased from 0.56 among low ketone producers and 3.36 among high ketone producers. The highest excretion of ketone bodies into the urine was $756 \mathrm{mmole} / \mathrm{g}$ creatinine. We suggested that the contribution of intestinal microbiota was also important [1113]. The involvement of intestinal microbiota may be a cause of the BHB individual difference. Our study clarified that hyperketonemia showed a correlation with pre fasting microbiota profile. 
High BHB level showed an association with family Enterobacteriaceae directly or indirectly. The dominance of Enterobacteriaceae seemed to suppress butyrate-producing bacteria. So, the BHB production seemed to be independent of butyrate pathway in the gut.

The increased BHB seemed to influence both mental and somatic symptoms.

BHB is reported to be produced in the liver, kidney, and astrocytes in the brain. Each organ would have its own chronological time in metabolism. Fasting would synchronize these gaps to improve the vivid and active feeling. The active and vivid feeling increased on the fourth day, and it lasted even one month later. The changes in mood were related to body change. Headache was the most popular symptom, as one-third female participants had a slight headache at the registry, but it remarkably improved by fasting. Hungry and GI distress, nausea appeared on the second and 3rd day, but these also improved toward the end.

Blood pressure was controlled by the complex network, and it was different by age category. In older age it seemed to be more influenced by body composition. Reset of many sensors inside the body by fasting would be an effective way to synchronize visceral time.

\section{Conclusion}

As the legacy effects of wellness fasting, we recognized improved lifestyles, normalized blood pressure, and increased diversity of intestinal microbiota. Metabolic changes inside the body should affect the chronomedical rhythm, and organ-specific chronological time could be harmonized by fasting. Dietary intervention is effective for the continuation of a good healthy state.

\section{Acknowledgement}

We thank the staffs of ARSOA wellness fasting and the contribution of participants as research volunteers. A part of this work was reported at the World Congress of Chronomedicine (WCC) 2018 held in Lucknow on 17-19 November 2018.

\section{Conflict of Interest}

The authors do not have COI with any companies. IU, SH, and RT are the employees of ARSOA group. Funding for this research was provided from the Lifescience Promoting Association.

\section{Bibliography}

1. Robinson AM and Williamson DH. "Physiological role of ketone bodies as substrates and signals in mammalian tissues". Physiology Review 60 (1980): 143-187.

2. Watanabe S., et al. "Basic ketone engine and booster glucose engine for energy production". Diabetes Research 2 (2016): $14-23$.
3. Heitmann RN., et al. "Hepatic ketogenesis and peripheral ketone body utilization in the nutrient". Journal of Nutrition 117 (1987): 1174-1180.

4. Newman JC and Verdin E. " $\beta$-hydroxybutyrate: Much more than a metabolite". Diabetes Research and Clinical Practice 106 (2014): 173-181.

5. Veech RL. "The therapeutic implications of ketone bodies: the effects of ketone bodies in pathological conditions: Ketosis, ketogenic diet, redox states, insulin resistance, and mitochondirial metabolism". Prostaglandins Leukot Essent Fatty Acids 70 (2004): 309-319.

6. Cornelissen G. Altered circadian energy metabolism and chronobiological risk factors of chronic diseases". In; The Role of Functional Food Security In Global Health.

7. Utada I., et al. "Wellness fasting for healthy longevity". Advances in Food Technology and Nutritional Sciences 2 (2016) :103-109.

8. Watanabe S. "Wellness fasting and hyperketosis". Diabetes Research 2 (2016): e10-e13.

9. Watanabe S., et al. "Ketone body production and excretion during wellness fasting". Diabetes Research 3 (2017): 1-8.

10. Koda M. "Science of the Fasting Therapy". Shunju-sha, Tokyo, Japan.

11. Hirakawa A., et al. "Koda's fasting therapy: Energy balance and intestinal bacteria flora". Advances in Food Technology and Nutritional Sciences 1 (2015): 112-123.

12. Watanabe S., et al. "Wellness Fasting-induced Hyperketosis and Interac-tion by Intestinal Microbiota". International Journal of Scientific and Engineering Research 8 (2017).

13. Watanabe S., et al. "Wellness fasting and intestinal Microbiota in chronomedicine". Acta Scientific Nutritional Health 3 (2019): 35-43.

Volume 3 Issue 8 August 2019

(C) All rights are reserved by Shaw Watanabe., et al. 